\title{
Large-Scale Cellulose-Assisted Transfer of Graphene Toward Industrial
}

\section{Applications}

\author{
Mingguang Chen ${ }^{\mathrm{a}, \mathrm{d}}$, Guanghui $\mathrm{Li}^{\mathrm{a}, \mathrm{d}}$, Wangxiang Li ${ }^{\mathrm{b}, \mathrm{d}}$, Dejan Stekovic ${ }^{\mathrm{b}, \mathrm{d}}$, Bassim Arkook ${ }^{\mathrm{c}, \mathrm{d}}$, \\ Mikhail E. Itkis ${ }^{a, b, c, d}$, Aron Pekker ${ }^{\text {b,d }}$, Elena Bekyarova ${ }^{\text {a,b,c,d,* }}$, Robert C. Haddon ${ }^{\text {a,b,c,d, * }}$ \\ a. Department of Chemical and Environmental Engineering, University of California, Riverside, \\ California 92521, United States. \\ b. Department of Chemistry, University of California, Riverside, California 92521, United States \\ c. Department of Physics, University of California, Riverside, California 92521, United States \\ d. Center for Nanoscale Science and Engineering, University of California, Riverside, California \\ 92521, United State
}

\begin{abstract}
CVD graphene has attracted a great deal of interest from both academia and industry. The strong motivation to commercialize high quality CVD graphene films and related devices has been restricted by the lack of a cheap, efficient, clean and reliable graphene transfer process. In this article, we report a novel graphene transfer technique which provides a route to high-throughput, reliable and economical transfer of graphene without introducing large cracks and residue contamination from polymers, such as PMMA or magnetic impurities. The transferred graphene was thoroughly characterized with Raman spectroscopy, Atomic Force Microscopy, and X-ray photoelectron spectroscopy. Fabricated large area graphene-based field effect transistors exhibited high mobilities, which were about 2 times higher than those for devices prepared with graphene transferred by the conventional wet transfer method. This new graphene transfer technique has the potential to expedite
\end{abstract}

*Corresponding author: Tel: 951 827-2450. E-mail: elenab@ucr.edu (Elena Bekyarova) 
the large scale industrial utilization of CVD graphene in electronics, spintronics, catalysis and energy storage.

\section{Introduction}

Graphene is a one atom thick two dimensional carbon material, whose valence and conduction bands touch each other at the Dirac point $[1,2]$. The fascinating properties, which originate from the unique electronic structure of graphene, motivate the wide interest in its potential application in many fields, such as electronics [3-8], spintronics [9], optics [5], environmental engineering [10, 11], and aerospace [12]. Chemical vapor deposition (CVD) is among the most promising methods for production of macrosize, continuous, high-quality graphene films for industrial applications [13-16].

The first step in the fabrication of devices based on CVD graphene is transfer of the as-grown graphene from copper or nickel to a targeted substrate, usually $\mathrm{SiO}_{2} / \mathrm{Si}$, boron nitride, or quartz. Although several transfer methods have been developed so far, few can be used for industrial applications due to their time-consuming, costly, or challenging scalability [17, 18]. A conventional graphene wet transfer method method [13, 14] widely employed in academia utilizes poly(methyl methacrylate) (PMMA) as the supporting layer and etching of the $\mathrm{Cu}$ or $\mathrm{Ni}$ substrate with iron chloride or iron nitrate [19-22]. However, researchers have been struggling with the degradation of graphene's intrinsic properties by contaminations from residues of PMMA and paramagnetic $\mathrm{Fe}^{3+}[23-25]$. Few advances have been made towards a high-throughput economic transfer of graphene for commercialization needs. Although the roll-to-roll (R2R) method can transfer large scale graphene onto targeted substrates $[16,26]$, the thermal release tape is known to leave contamination on the graphene layer $[14,27]$ and $\mathrm{R} 2 \mathrm{R}$ method is not applicable to $\mathrm{SiO}_{2} / \mathrm{Si}$ substrates, limiting its application in the semiconductor industry. The face-to-face graphene transfer method offers large-area, continuous graphene films with reduced density of transfer defects, however, the process is designed for "stiff" substrates [17]. The clean lifting transfer (CLT) method solved the issue of PMMA residue by making use of an electrostatic attraction force to protect graphene during the transfer process. However, this 
method uses high voltage and it has a strict requirement for the surface flatness of the as-grown graphene on $\mathrm{Cu}$ or $\mathrm{Ni}$, which restrict its potential industrial application. Additionally, the contamination with paramagnetic $\mathrm{Fe}^{3+}$ remains an unresolved issue. For transfer methods that employed ammonium persulfate as the etching solution in order to avoid contamination from paramagnetic materials [28-30], the cost and time increased dramatically.

In this article, we discuss the development of a novel technique for the transfer of graphene on various substrates. The method utilizes cellulose acetate as the coating layer, which protects graphene from unfavourable forces and contaminations during the transfer process by forming a soft flexible thin film on top of graphene. The etching of $\mathrm{Cu}$ foil, used in the CVD graphene growth, is accomplished with a hydrogen peroxide - hydrogen chloride solution, which decreases the possibility for magnetic contamination of the graphene. This cellulose assisted transfer (CAT) method largely reduces the cost, time and contaminations of the obtained graphene layer and can be expanded to industrial scale applications. The main advantages of the CAT method in comparison with other graphene transfer techniques are summarized in Table 1. The transferred grapene films were characterized with Raman spectroscopy, atomic force microscopy (AFM) and X-ray photoelectron spectroscopy (XPS) to evaluate the quality of the obtained films. The fabricated field effect transistor (FET) devices showed high mobility and on-off ratio.

Table1 Comparison of several parameters of different graphene transfer methods.

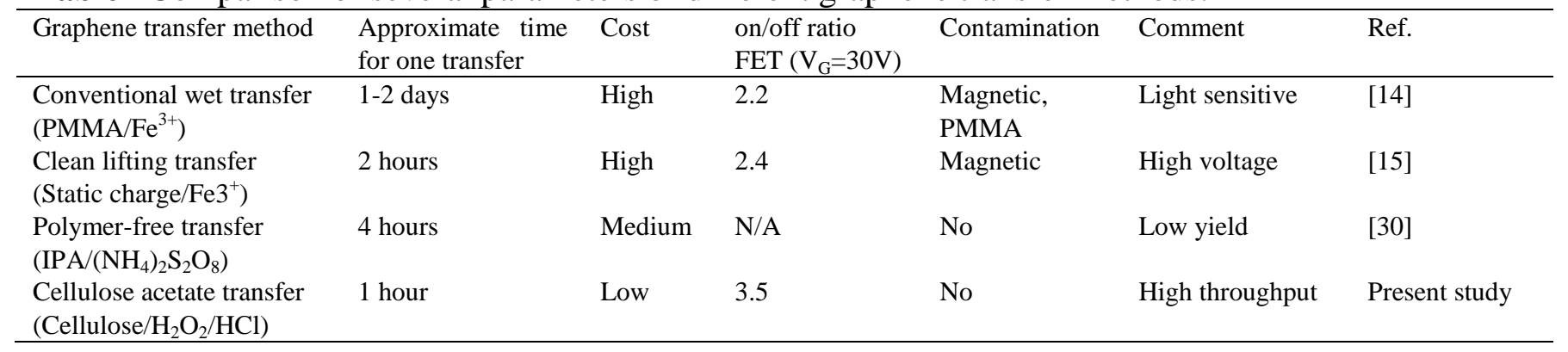




\section{Experimental}

\subsection{CAT graphene transfer}

As-grown monolayer CVD graphene (Graphene Supermarket) was first spin coated with a cellulose acetate solution (Average Mn 50,000 by GPC, Sigma Aldrich, $4.5 \mathrm{mg} / \mathrm{mL}$ dissolved in acetone) at $2000 \mathrm{rpm}$. The spin-coated thin film dries immediately as the acetone evaporates. The graphene on the uncoated side was etched away by oxygen plasma (Oxford Plasmalab 100/180 model; forward power 30 W; ICP power $300 \mathrm{~W}$, etching time $30 \mathrm{~s}$ ). An aqueous solution of $\mathrm{H}_{2} \mathrm{O}_{2}$ and $\mathrm{HCl}$ was prepared by mixing equal volumes of $2 \mathrm{M} \mathrm{HCl}$ and $1 \mathrm{M} \mathrm{H}_{2} \mathrm{O}_{2}$; the solution etches away the $\mathrm{Cu}$ substrate $(25 \mu \mathrm{m})$ underneath the CVD graphene within 10 minutes. The graphene sample was thoroughly cleaned by replacing the etching solution with distilled water. The substrate with the transferred graphene was placed on a hot plate and kept at $35^{\circ} \mathrm{C}$ for $10 \mathrm{~min}$, followed by baking at $200{ }^{\circ} \mathrm{C}$ for $20 \mathrm{~min}$. After the substrate with the graphene was cooled down to $\sim 60{ }^{\circ} \mathrm{C}$, it was submerged into a hot acetone bath overnight to remove the cellulose acetate.

\subsection{PMMA graphene transfer}

As-grown monolayer CVD graphene was first spin coated with a PMMA solution (950 PMMA A4, MicroChem, $10 \%$ by volume dissolved in chlorobenzene) at $2000 \mathrm{rpm}$. The spin-coated thin film was kept at room temperature overnight inside a clean petri dish. The graphene on the uncoated side was etched away by oxygen plasma as described in section 2.1. Then the PMMA/Graphene/Cu sample was placed on the surface of $\mathrm{FeCl}_{3}$ solution $(0.5 \mathrm{M})$ to etch away the $\mathrm{Cu}$ substrate (thickness $25 \mu$ m; typical etching time 60 minutes). After the $\mathrm{Cu}$ was removed, the etching solution was replaced with clean water by repeatedly flowing out the waste solution and flowing in clean water until neutral $\mathrm{pH}$ was reached. The substrate with the graphene was transferred to a hot plate and kept at $35^{\circ} \mathrm{C}$ for $10 \mathrm{~min}$. After this step the temperature was increased and maintained at $200{ }^{\circ} \mathrm{C}$ for 20 minutes. The substrate with the graphene was cooled down to $\sim 60{ }^{\circ} \mathrm{C}$ and kept in a hot acetone bath overnight to remove the PMMA. 


\subsection{Raman spectroscopy and mapping}

Raman spectra and mapping of CVD graphene were recorded with a Nicolet Almega XR Dispersive Raman microscope using laser excitation of $532 \mathrm{~nm}$ at $25 \%$ power. The laser spot size is around $1 \mu \mathrm{m}$ with a $50 \mathrm{x}$ objective lens. The Raman mapping area for all samples is $20 \mu \mathrm{m} \times 18 \mu \mathrm{m}$, with a step size of $1 \mu \mathrm{m}$ in both $x$ and $y$ directions.

\subsection{Atomic Force Microscopy (AFM)}

AFM images were collected in a tapping mode with Digital Instruments, Nanoscope IIIA.

\section{$2.5 X$-ray photoelectron spectroscopy (XPS)}

XPS of transferred CVD graphene on $300 \mathrm{~nm} \mathrm{SiO}_{2} / \mathrm{Si}$ substrates was carried out with a Kratos AXIS ULTRADLD XPS system equipped with Al Ka monochromated X-ray source and a $165 \mathrm{~mm}$ mean radius electron energy hemispherical analyzer. Vacuum pressure was kept below $3 \times 10^{-9}$ torr during the acquisition. The high-resolution scans were run using a power of $300 \mathrm{~W}, 20$ pass energy and a step size of $0.05 \mathrm{eV}$. A low-energy electron flood from a filament was used for charge neutralization. The size slot for the XPS is $300 \mu \mathrm{m} \times 700 \mu \mathrm{m}$. The peak fits consist of Lorentzian and Gaussian distributions.

\subsection{Fabrication of field effect transistors (FETs)}

Drain and source electrodes $(10 \mathrm{~nm} \mathrm{Cr} / 100 \mathrm{~nm} \mathrm{Au})$ were evaporated by an Ebeam Evaporator onto $300 \mathrm{~nm} \mathrm{SiO}_{2} / \mathrm{Si}$ substrates with a shadow mask. After that, as-grown monolayer CVD graphene was transferred onto the pre-patterned substrates by conventional PMMA transfer and CAT methods, respectively. The channel dimensions of all graphene devices are $0.25 \mathrm{~mm}$ (length) x $1 \mathrm{~mm}$ (width). No photolithography was employed to exclude the effect of photoresist residues on the electronic properties

of the transferred graphene samples. Thermal annealing at $200{ }^{\circ} \mathrm{C}$ in vacuum $\left(5 \times 10^{-7}\right.$ torr $)$ for 2 hours was performed to remove atmospheric dopants and bring the Dirac point in the vicinity of 0 volts. 


\section{Results and Discussion}

\subsection{Description of the graphene transfer method}

In this work, we employed cellulose acetate as a holding layer to protect graphene during the transfer process because of its low cost, excellent draping qualities and environmentally friendly properties. Cellulose acetate, an acetylated derivative of the naturally abundant and sustainable polymer cellulose, can be dissolved in acetone and, when spin-coated on a flat surface, it gives a continuous thin and flexible film (Fig. 1). Cellulose acetate has a long history of application in a number of industrial fields. It has been used as the film base in photography [31], as a substrate for magnetic tape [32], and as a component in household fabrics [33], among other applications. Thus, a mature and comprehensive system to produce, transport and stock cellulose acetate has already been built.

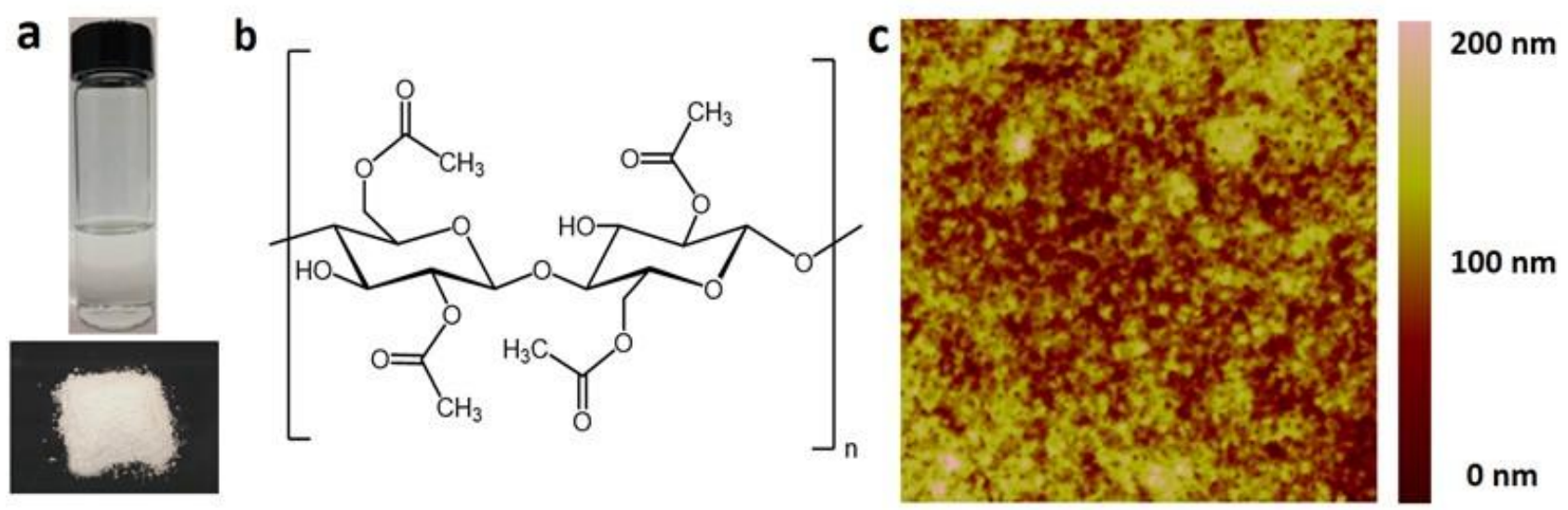

Fig. 1 (a) Photographs of a cellulose acetate powder (bottom) and a cellulose acetate solution in acetone (top). (b) Structure of cellulose acetate. (c) AFM image of a spin-coated cellulose acetate thin film on top of as-grown CVD graphene.

For the graphene transfer, a thin layer of cellulose acetate film is first spin-coated on top of the graphene film grown on $\mathrm{Cu}$ or $\mathrm{Ni}$, as shown in Fig. 2a. The cellulose acetate film adheres strongly to the graphene surface and protects it from the surface tension of the etching solution after the $\mathrm{Cu}$ or $\mathrm{Ni}$ are removed. The metal substrate $(\mathrm{Cu})$ is etched with an $\mathrm{H}_{2} \mathrm{O}_{2} / \mathrm{HCl} / \mathrm{H}_{2} \mathrm{O}$ solution. For the etching the central 
batch reactor (Fig. 2) is filled with a fresh $\mathrm{H}_{2} \mathrm{O}_{2} / \mathrm{HCl} / \mathrm{H}_{2} \mathrm{O}$ solution and the cellulose acetate/graphene/copper sample is placed on the surface of the etching solution. The etching occurs through the following chemical reaction:

$$
\mathrm{H}_{2} \mathrm{O}_{2}+2 \mathrm{HCl}+\mathrm{Cu} \rightarrow \mathrm{CuCl}_{2}+2 \mathrm{H}_{2} \mathrm{O}
$$

After the copper is completely etched away, fresh water is introduced into the central reactor from the inlet tank and the waste solution is extracted from the outlet tank at the same rate using peristaltic pumps, thus converting the system into a continuous tank reactor. After the $\mathrm{pH}$ of the solution inside the reactor approaches that of pure water, the target substrate is positioned onto the bottom of the central reactor through a slit designed between the inlet tank and the central reactor. In the next step, the water is drained out from the outlet tank at a slow rate allowing the cellulose acetate/graphene to lower down onto the substrate.

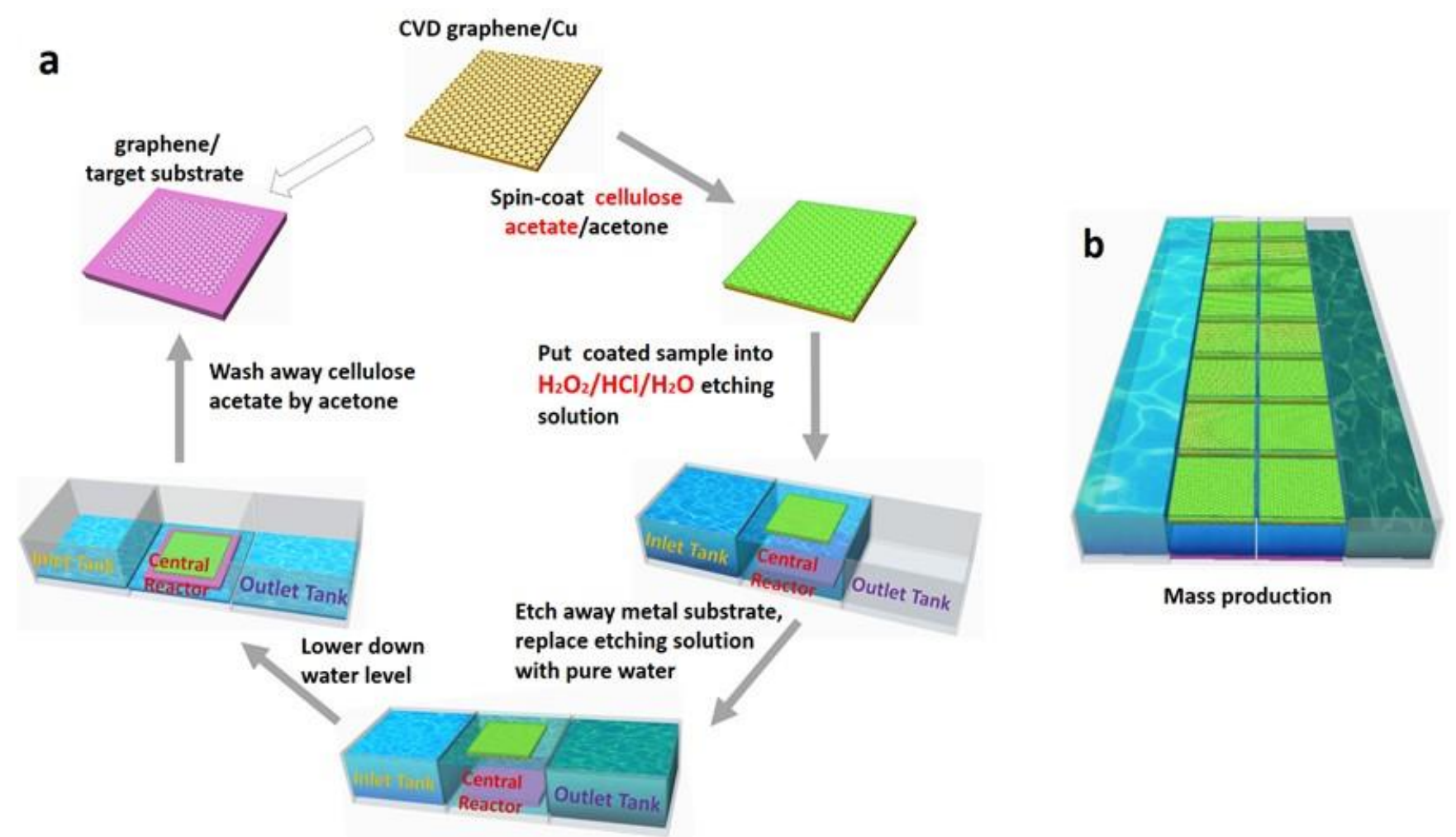

Fig. 2 Schematic illustration of (a) the CAT method and (b) the reactor for simultaneous transferring graphene onto multiple substrates for industrial applications. 
Alternatively, the floating cellulose acetate coated graphene can be lifted out from the pure water bath with the target substrate. Next, the cellulose acetate/graphene/target substrate is transferred to a hot plate and kept at $35{ }^{\circ} \mathrm{C}$ for 10 min to remove large water droplets trapped between the graphene film and the substrate. The temperature is then increased to $200{ }^{\circ} \mathrm{C}$ and maintained for $20 \mathrm{~min}$ to enhance the contact between graphene and the substrate. The final step of the transfer process is removing the cellulose film, which is dissolved in hot acetone vapors for 5 minutes. The substrate with the graphene is then submerged into hot acetone to completely remove the cellulose acetate. The process can be easily scaled to simultaneously conduct the graphene transfer on multiple substrates as illustrated in Fig. 2 b.

\subsection{Characterization of the transferred graphene}

In order to systematically investigate the quality of the CAT graphene and compare it with the conventional wet transfer method, various characterization tools were employed. Raman spectroscopy is a powerful and sensitive technique to characterize the quality of carbon materials, specifically graphene $[34,35]$. The appearance of a D peak in the vicinity of $1340 \mathrm{~cm}^{-1}$ generally indicates presence of defects in the $\mathrm{sp}^{2}$ carbon network of graphene $[34,36]$. Other important metrics are the amplitude and spectral shape of the $G$ and $2 D$ peaks, observed in the vicinity of $1580 \mathrm{~cm}^{-1}$ and $2700 \mathrm{~cm}^{-1}$, respectively, which can be utilized to characterize the quality of graphene and the number of graphene layers [35]. For example, because the $2 \mathrm{D}$ peak is sensitive to long range order, higher intensity of the $2 \mathrm{D}$ peak is an indication of higher crystalline quality of the single layer graphene [35]. Fig. 3a compares representative Raman spectra of graphene samples transferred by the CAT and PMMA-assisted methods. The CAT graphene typically gives a Raman spectrum with little or no D-peak as compared to the obvious D-peak present in the Raman spectrum of graphene after the PMMA transfer. This is an indication of a much smaller concentration of defects in the CAT-graphene. G- and 2D-peaks are well pronounced in both samples, but the $2 \mathrm{D}$ to $\mathrm{G}$ peak ratio is approximately 2 times higher for the CAT graphene film 
confirming its higher quality as compared to the conventional wet transfer method. Moreover, the slight blue shift of the position of $\mathrm{G}$ peak and 2D peak of PMMA-graphene compared to CAT-graphene indicates that $\mathrm{p}$ type dopants were introduced [37, 38], most likely due to PMMA residues [29].
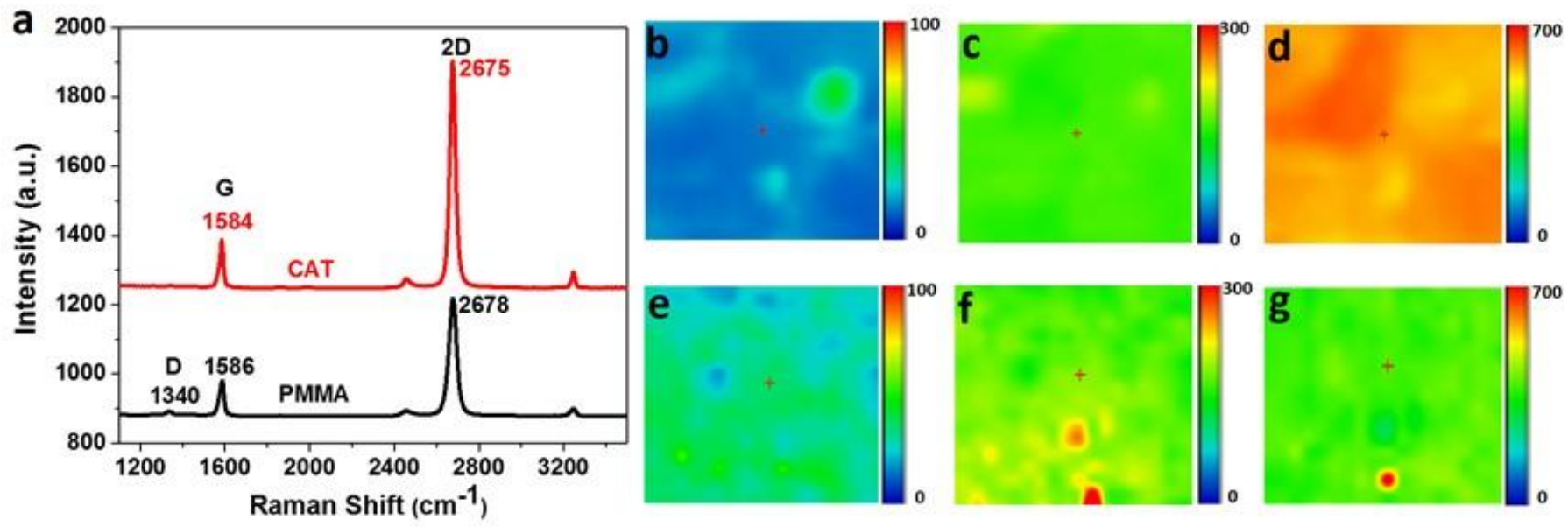

Fig. 3 (a) Raman spectra of single layer CVD graphene transferred by the CAT and PMMA methods. (b-d) Raman intensity maps of D-, G- and 2D- peaks, respectively, for CAT-graphene on a $\mathrm{SiO}_{2} / \mathrm{Si}$ substrate. (e-g) Raman intensity maps of D-, G- and 2D- peaks, respectively, for PMMA-graphene on a $\mathrm{SiO}_{2} / \mathrm{Si}$ substrate.

In addition to the evaluation of the individual spectra (Fig. 3a) we conducted a statistical comparison of the quality of the graphene films by collecting Raman maps of $20 \mu \mathrm{m} \times 18 \mu \mathrm{m}$ areas. The $\mathrm{D}, \mathrm{G}$ and 2D peak maps are shown in Fig. 3b-3d for the CAT-graphene and Fig. 3e-3g for the PMMA transferred graphene, respectively. Comparison of the D-peak maps (Fig.3b and 3e) shows that most of the area in the case of CAT-graphene is defect free and less than $10 \%$ of the area displays a relatively small D peak, which confirms that the CAT method yields graphene with significantly lower defect density as compared to the conventional wet transfer method. Fig. 3c and $3 \mathrm{f}$ show similar amplitudes of the $\mathrm{G}$ peak across both graphene samples, while the average amplitude of the $2 \mathrm{D}$ peak in CAT-graphene sample is about 2 times higher than that in PMMA-graphene, which indicates that the CAT method preserves the quality of single layer CVD graphene. Overall, Raman spectroscopy shows that the CAT-graphene is 
almost defect free (Fig. 3b), displaying a relatively uniform large intensity 2D peak (Fig. 3d), while the PMMA-transferred graphene displays a prominent D peak throughout the mapped surface (Fig. 3e) accompanied with a lower intensity 2D peak (Fig. 3g).

Atomic force microscopy (AFM) was employed to characterize the surface cleanliness and morphology of the graphene transferred by both methods. As shown in Fig. 4a, graphene transferred by the CAT method shows a very clean and relatively uniform surface with an average roughness ( $\mathrm{Ra}$ ) of $0.4 \mathrm{~nm}$. The AFM images revealed the presence of wrinkles with an average height of $\sim 1 \mathrm{~nm}$ (Fig. 4b). The majority of the wrinkles presumably originate from the growth process of CVD graphene (formed during the cool down step) [13]. Some wrinkles may be introduced during the transfer process by mechanical manipulation or thermal expansion due to temperature change. We note that even scotch tape exfoliated graphene may have wrinkles [39]. In contrast, the surface of the PMMA-assisted graphene is covered with a polymer residue (Fig. 4d) with a typical height of the impurities being $>5$ $\mathrm{nm}$ and an average roughness of $1 \mathrm{~nm}, 2.5$ times higher than in CAT-graphene (Fig. 4e). The cleanness of the CAT-graphene is further confirmed by XPS spectroscopy. Fig. 4g,h display the typical C1s core spectra of graphene samples transferred by the CAT and PMMA methods. The CAT-graphene gives rise to a narrow $\mathrm{C} 1 \mathrm{~s}$ peak with a major contribution from $\mathrm{sp}^{2}$ hybridized $\mathrm{C}$ atoms $(284.4 \mathrm{eV})$ and a shoulder at $285.3 \mathrm{eV}$ associated with $\mathrm{sp}^{3}$ carbon presumably due to edges and defects [23]. The $\mathrm{C} 1 \mathrm{~s}$ spectrum of the PMMA transferred graphene is broad and it can be deconvoluted to five peaks. In addition to the $\mathrm{sp}^{2}$ hybridized $(284.4 \mathrm{eV})$ and $\mathrm{sp}^{3}$ hybridized $(285.1 \mathrm{eV})$ carbon peaks, there is a significant contribution from $\mathrm{C}-\mathrm{O}$ species associated with the PMMA residue $(\mathrm{C}-\mathrm{O}$ at $286.5 \mathrm{eV}, \mathrm{C}=\mathrm{O}$ at $287.5 \mathrm{eV}$ and $\mathrm{C}-\mathrm{C}=\mathrm{O}$ at $289 \mathrm{eV})[23]$. 

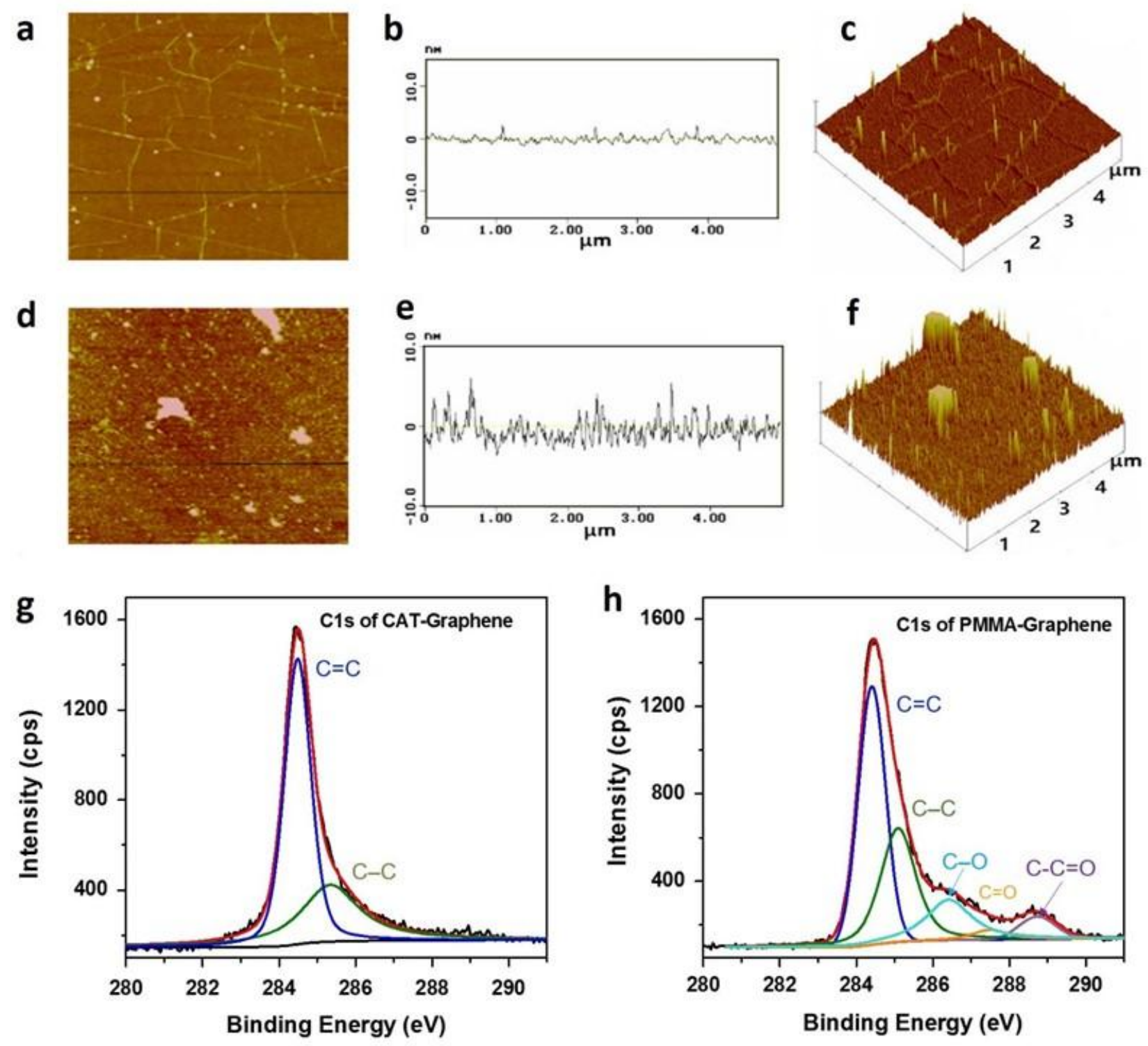

Fig. 4 AFM images (amplitude, height profile and 3D-morphology) of a single layer CVD graphene on $300 \mathrm{~nm} \mathrm{SiO} 2 / \mathrm{Si}$ transferred by (a-c) CAT method (d-f) PMMA method. C1s core spectra of single layer graphene transferred by (g) CAT and (h) PMMA method. The peak fits consist of Lorentzian and Gaussian distributions.

In order to compare the electronic transport properties of the CVD graphene prepared by the CAT and PMMA transfers, graphene-based back gated field effect transistors (FETs) were fabricated on $300 \mathrm{~nm}$ $\mathrm{SiO}_{2} / \mathrm{Si}$ substrates with pre-patterned $\mathrm{Cr} / \mathrm{Au}$ electrodes. Relatively large dimensions of graphene devices 
with a channel length of $0.25 \mathrm{~mm}$ and channel width of $1 \mathrm{~mm}$ were fabricated in order to avoid contamination with resist residues during the photolithography or the e-beam lithography processes. Prior to measurements the devices were annealed at $200{ }^{\circ} \mathrm{C}$ in vacuum for 2 hours. Fig. 5a shows the transport characteristics of the corresponding FET devices. The FET fabricated with the CAT-graphene shows sharper and more symmetric transport characteristics in comparison with the PMMA-transferred graphene device. The hole and electron mobilities extracted from the linear range for CAT transferred graphene are $1695 \mathrm{~cm}^{2} \mathrm{~V}^{-1} \mathrm{~s}^{-1}$ and $1675 \mathrm{~cm}^{2} \mathrm{~V}^{-1} \mathrm{~s}^{-1}$, respectively, significantly higher than those obtained for the PMMA transferred graphene $\left(450 \mathrm{~cm}^{2} \mathrm{~V}^{-1} \mathrm{~s}^{-1}\right.$ for holes and $380 \mathrm{~cm}^{2} \mathrm{~V}^{-1} \mathrm{~s}^{-1}$ for electrons).
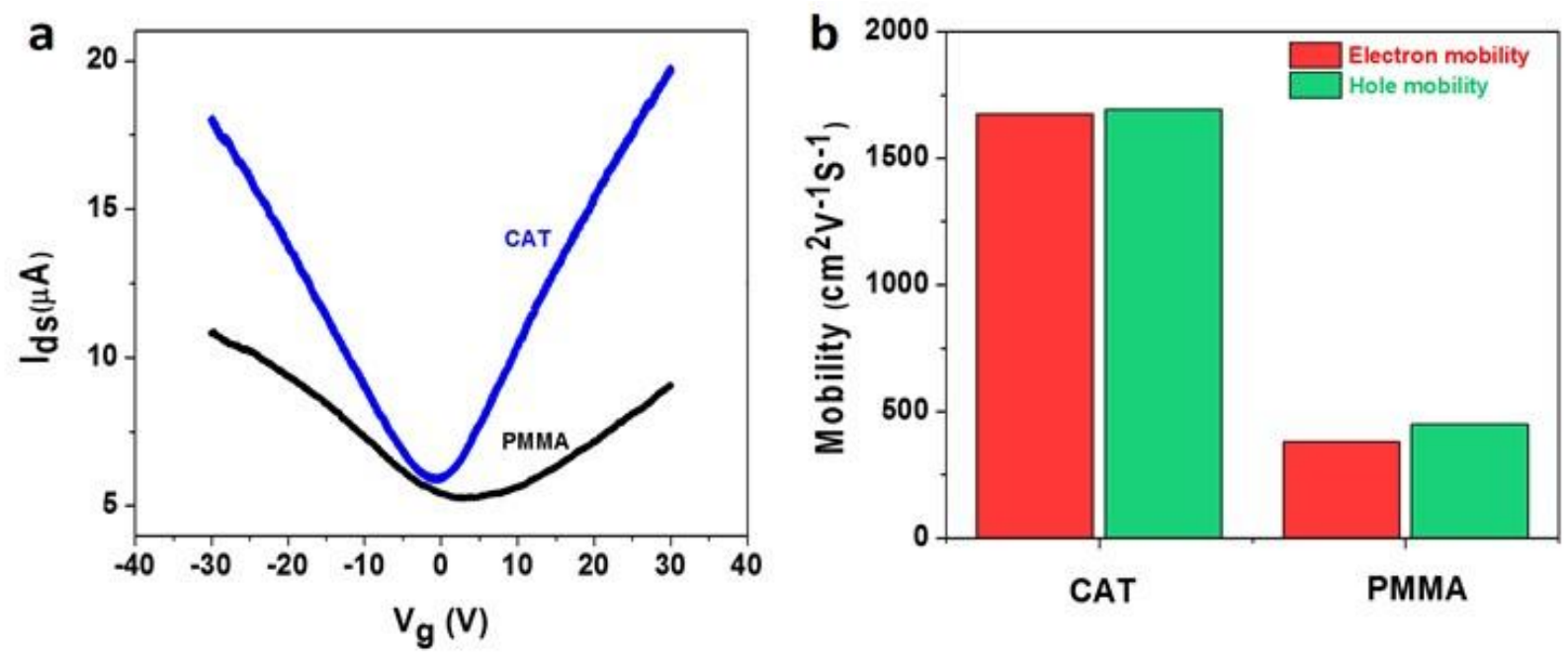

Fig. 5 FET characterization of graphene transferred onto $300 \mathrm{~nm} \mathrm{SiO} / 2 \mathrm{Si}$ substrates. (a) Drain-source current vs gate voltage of the FET devices. (b) Comparison of the mobilities of the large size CATgraphene and PMMA-graphene devices.

The larger difference between the hole and electron mobilities for the PMMA transferred graphene device indicates an asymmetry of scattering for the two types of carriers, which may be caused by the residual contaminants $\left(\mathrm{Fe}^{3+}\right.$ and PMMA). In principle, higher mobility values were reported in the literature for some CVD graphene samples [13],[17], however those reports typically utilize graphene 
channels of microscopic $(<10 \mu \mathrm{m})$ dimensions, while the current study was conducted with millimeter scale devices in which high mobility is much more difficult to achieve.

Compared with the PMMA transferred method, the cellulose assisted transfer introduces much fewer residues, and magnetic impurities, which largely conserves the transport properties of the original CVD graphene and thus obtain higher mobility devices.

\section{Conclusion}

In summary, a novel cellulose acetate assisted graphene transfer method (CAT) suitable for both academic and industrial applications is presented in this paper. The CAT system not only solves the problems associated with PMMA and magnetic material contaminations introduced by most transfer methods, but also largely reduces the transfer cost and time, while providing a very convenient system for precise and efficient transfer of clean graphene on various substrates. We demonstrated the advantages and high quality of the CAT- graphene by means of Raman spectroscopy, Atomic Force Microscopy, and X-ray photoelectron spectroscopy. Macroscopic size single-layer graphene-based field effect transistors were fabricated and the extracted mobilities were about 2 times higher for the CATgraphene devices than those for the PMMA-graphene devices. This method has potential to facilitate graphene related research and expedite the commercialization of CVD graphene.

\section{Acknowledgements}

This work was funded by NSF under contract DMR-1305724. XPS measurements were performed with an instrument acquired with NSF support under contract DMR-0958796. We would like to thank Dr. Ruoxue Yan for valuable discussions.

\section{References}

[1] PR Wallace. The band theory of graphite. Phys Rev 71(9) (1947) 622-34.

[2] KS Novoselov, AK Geim, SV Morozov, D Jiang, Y Zhang, SV Dubonos, et al. Electric field effect in atomically thin carbon films. Science. 306 (2004) 666-9. 
[3] C Berger, Z Song, T Li, X Li, AY Ogbazghi, R Feng, et al. Ultrathin epitaxial graphite: 2D electron gas properties and a route toward graphene-based nanoelectronics. J Phys Chem B. 108(52) (2004) 19912-6.

[4] ZH Chen, YM Lin, MJ Rooks, P Avouris. Graphene nano-ribbon electronics. Physica E. 40(2) (2007) 228-32.

[5] C-H Liu, Y-C Chang, TE Norris, Z Zhong. Graphene photodetectors with ultra-broadband and high responsivity at room temperature. Nature Nanotech. 9 (2014) 273-8.

[6] F Torrisi, T Hasan, WP Wu, ZP Sun, A Lombardo, TS Kulmala, et al. Inkjet-printed graphene electronics. ACS Nano. 6(4) (2012) 2992-3006.

[7] E Bekyarova, S Niyogi, S Sarkar, X Tian, M Chen, ML Moser, et al. Stereochemical effect of covalent chemistry on the electronic structure and properties of the carbon allotropes and graphene surfaces. Synth Met. 210 (2015) 80-4.

[8] M Chen, X Tian, W Li, E Bekyarova, G Li, M Moser, et al. Application of organometallic chemistry to the electrical interconnection of graphene nanoplatelets. Chem Mater. 28(7) (2016) 2260-6.

[9] RR Nair, M Sepioni, I-L Tsai, PO Lehtinen, J Keinonen, AV Krasheninnikov, et al. Spin-half paramagnetism in graphene induced by point defects. Nature Phys. 8(2012) 199-202.

[10] Y Li, H Yuan, A von dem Bussche, M Creighton, RH Hurt, AB Kane, et al. Graphene microsheets enter cells through spontaneous membrane penetration at edge asperities and corner sites. Proc Natl Acad Sci USA. 110(30) (2013) 12295-300.

[11] F Guo, G Silverberg, S Bowers, S Kim, D Datta, V Shenoy, et al. Graphene-based environmental barriers. Environ Sci Technol. 46(14) (2012) 7717-24.

[12] T Zhang, H Chang, Y Wu, P Xiao, N Yi, Y Lu, et al. Macroscopic and direct light propulsion of bulk graphene material. Nat Photonics. 9(7) (2015) 471-6.

[13] XS Li, WW Cai, JH An, S Kim, J Nah, DX Yang, et al. Large-area synthesis of high-quality and uniform graphene films on copper foils. Science. 324(5932) (2009) 1312-4.

[14] X Liang, BA Sperling, I Calizo, G Cheng, CA Hacker, Q Zhang, et al. Toward clean and crackless transfer of graphene. ACS Nano. 5(11) (2011) 9144-53.

[15] D Wang, I Huang, P Ho, S Li, Y Yeh, D Wang, et al. Clean-lifting transfer of large-area residualfree graphene films. Adv Mater. 25(32) (2013) 4521-6.

[16] T Kobayashi, M Bando, N Kimura, K Shimizu, K Kadono, N Umezu, et al. Production of a 100$\mathrm{m}$-long high-quality graphene transparent conductive film by roll-to-roll chemical vapor deposition and transfer process. Appl Phys Lett. 102(2) (2013) 023112. 
[17] L Gao, GX Ni, YP Liu, B Liu, AH Castro Neto, KP Loh. Face-to-face transfer of wafer-scale graphene films. Nature. 505(7482) (2014) 190-4.

[18] SJ Kim, T Choi, B Lee, S Lee, K Choi, J Park, et al. Ultraclean patterned transfer of single-layer graphene by recyclable pressure sensitive adhesive films. Nano Lett. 15(5) (2015) 3236-40.

[19] XS Li, YW Zhu, WW Cai, M Borysiak, BY Han, D Chen, et al. Transfer of large-area graphene films for high-performance transparent conductive electrodes. Nano Lett. 9(12) (2009) 4359-63.

[20] Y Wu, Y Lin, AA Bol, KA Jenkins, F Xia, DB Farmer, et al. High-frequency, scaled graphene transistors on diamond-like carbon. Nature. 472(7341) (2011) 74-8.

[21] Q Yu, LA Jauregui, W Wu, R Colby, J Tian, Z Su, et al. Control and characterization of individual grains and grain boundaries in graphene grown by chemical vapour deposition. Nature Mater. 10(6) (2011) 443-9.

[22] Y Wu, KA Jenkins, A Valdes-Garcia, DB Farmer, Y Zhu, AA Bol, et al. State-of-the-art graphene high-frequency electronics. Nano Lett. 12(6) (2012) 3062-7.

[23] Y Lin, C Lu, C Yeh, C Jin, K Suenaga, P Chiu. Graphene annealing: How clean can it be? Nano Lett. 12(1) (2011) 414-9.

[24] H Park, PR Brown, V Bulovic, J Kong. Graphene as transparent conducting electrodes in organic photovoltaics: Studies in graphene morphology, hole transporting layers, and counter electrodes. Nano Lett. 12(1) (2012) 133-40.

[25] A Ambrosi, M Pumera. The cvd graphene transfer procedure introduces metallic impurities which alter the graphene electrochemical properties. Nanoscale. 6(1) (2014) 472-6.

[26] S Bae, H Kim, Y Lee, XF Xu, JS Park, Y Zheng, et al. Roll-to-roll production of 30-inch graphene films for transparent electrodes. Nat Nanotech. 5(8) (2010) 574-8.

[27] Y Lee, K Tu, C Yu, S Li, J Hwang, C Lin, et al. Top laminated graphene electrode in a semitransparent polymer solar cell by simultaneous thermal annealing/releasing method. ACS Nano. 5(8) (2011) 6564-70.

[28] JW Suk, A Kitt, CW Magnuson, Y Hao, S Ahmed, J An, et al. Transfer of CVD-grown monolayer graphene onto arbitrary substrates. ACS Nano. 5(9) (2011) 6916-24.

[29] A Pirkle, J Chan, A Venugopal, D Hinojos, CW Magnuson, S McDonnell, et al. The effect of chemical residues on the physical and electrical properties of chemical vapor deposited graphene transferred to $\mathrm{SiO}_{2}$. Appl Phys Lett. 99(12) (2011) 122108.

[30] WH Lin, TH Chen, JK Chang, JI Taur, YY Lo, WL Lee, et al. A direct and polymer-free method for transferring graphene grown by chemical vapor deposition to any substrate. ACS Nano. 8(2) (2014) 1784-91. 
[31] JS Machell, ID Sand, inventors; Light sensitive silver halide element with cellulose ester film base. US patent US5288715 A. 1994.

[32] BRA Von, inventor Magnetic recording tape and method of making same. US patent US 2711901 A. 1955.

[33] JC Chen, KJ Soden, inventors; Anti-adhesion cellulose acetate wound dressing. US patent US 6500539 B1. 2002.

[34] MS Dresselhaus, A Jorio, M Hofmann, G Dresselhaus, R Saito. Perspectives on carbon nanotubes and graphene raman spectroscopy. Nano Lett. 10(3) (2010) 751-8.

[35] AC Ferrari, DM Basko. Raman spectroscopy as a versatile tool for studying the properties of graphene. Nature Nanotech. 8(4) (2013) 235-46.

[36] AC Ferrari, JC Meyer, V Scardaci, C Casiraghi, M Lazzeri, F Mauri, et al. Raman spectrum of graphene and graphene layers. Phys Rev Lett. 97(2006) 187401-4.

[37] S Pisana, M Lazzeri, C Casiraghi, KS Novoselov, AK Geim, AC Ferrari, et al. Breakdown of the adiabatic born-oppenheimer approximation in graphene. Nature Mater. 6(2007) 198-201.

[38] A Das, S Pisana, B Chakraborty, S Piscanec, SK Saha, UV Waghmare, et al. Monitoring dopants by Raman scattering in an electrochemically top-gated graphene transistor. Nature Nanotech. 3(2008) 210-5.

[39] SV Morozov, KS Novoselov, MI Katsnelson, F Schedin, LA Ponomarenko, D Jiang, et al. Strong suppression of weak localization in graphene. Phys Rev Lett. 97 (2006) 016801. 


\section{CVD graphene/Cu}

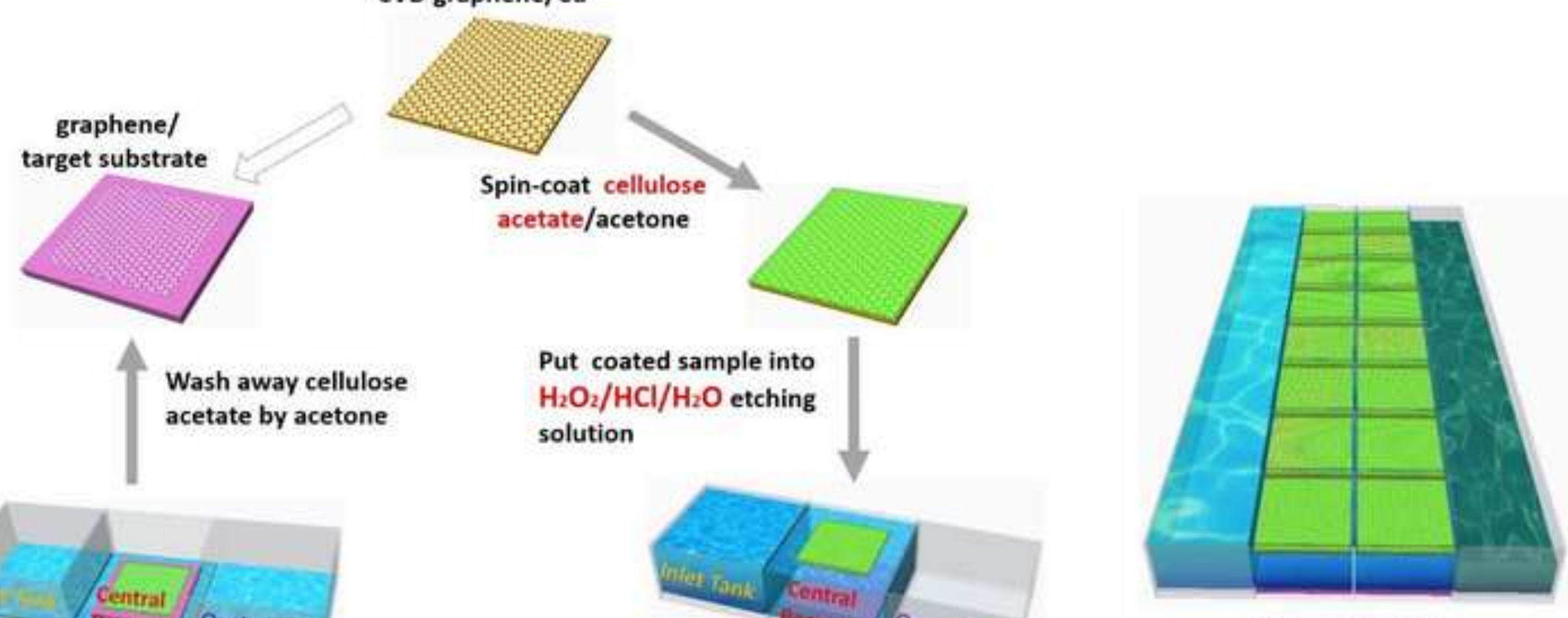

Etch away metal substrate,

Mass production replace etching solution

with pure water

Lower down

water level

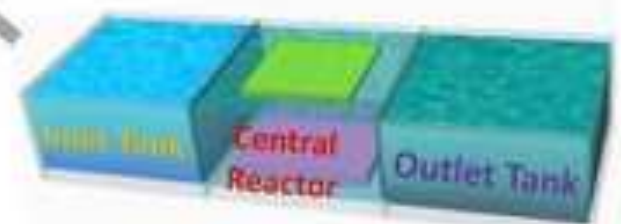

